[ARTICLE]

\title{
THE PRESENT PROGRESSIVE WITH FUTURE TIME REFERENCE VS. BE GOING TO: IS DOC BROWN GOING BACK TO THE FUTURE BECAUSE HE IS GOING TO RECONSTRUCT IT?
}

\author{
NAOAKI WADA \\ University of Tsukuba
}

\begin{abstract}
This paper, based on the tense theory presented in Wada (2001a), offers a comparative study of differences and similarities between sentences containing the present progressive with future time reference and those containing be going to. The reason for adopting this theory is not only because it has provided systematic explanations for several English tense phenomena, but also because it has analyzed the mechanism of interpreting sentences containing other future time expressions such as will and the simple present with future time reference. It is shown that the temporal structures of sentences containing the present progressive and be going to provide a basis for explaining the differences and similarities between their tense phenomena.*
\end{abstract}

Keywords: temporal structure, present progressive, be going to, preliminary stage, future

\section{Introduction}

There have been many studies on comparisons between will and be going to (Binnick (1971, 1972), Brisard (2001), Coates (1983), Haegeman (1989), McIntosh (1966), Nicolle (1998), Wada (1996, 2001a), Wekker (1976)), on one hand, and comparisons between the simple present and the present progressive with future time reference (Hirtle and Curat (1986), Huddleston (1977), Prince (1982), Smith (1981), Wekker (1976)), on the other. However, little attention has been paid to comparisons between be going to and the present progressive with future time reference-usually referred to as the futurate progressive-, which are similar in that they are both said to

* I would like to thank Yukio Hirose, Junya Watanabe, and three anonymous EL reviewers for their comments and stylistic suggestions on earlier versions of this paper. I would also thank James Andrew Elwood for improving my English. All remaining inadequacies are my own. This work is supported by Grant-in-Aids for Scientific Research from the Ministry of Education, Culture, Sports, Science and Technology, Grant No. 19320070 and Grant No. 19520414.

English Linguistics 26: 1 (2009) 96-131 -96-

(C) 2009 by the English Linguistic Society of Japan 
represent the near future, assured future, and present-orientation. As far as I know, only a few grammar books provide simple accounts of comparisons between sentences containing be going to (BGT-sentences) and those containing the present progressive with future time reference (PPF-sentences); there are no systematic comparative studies of them.

This paper aims to provide a comparative study of PPF-sentences and BGT-sentences on the basis of a systematic theory of tense. ${ }^{1}$ To this end, the present analysis is based on the tense theory proposed in Wada (2001a). This theory not only has provided systematic explanations for several English tense phenomena, but also has analyzed differences between sentences containing will (W-sentences) and BGT-sentences, on one hand, and the mechanism of interpreting sentences containing the simple present with future time reference (SPF-sentences)-usually referred to as the simple futurate-, on the other (cf. Wada (2001b)). ${ }^{2}$ Therefore, a comparative study within this framework will provide not only a systematic analysis of differences and similarities between PPF- and BGT-sentences, but also a more convincing explanation of them in that the two sentence types can potentially be analyzed in comparison with other future time expressions such as W- and SPF-sentences.

This paper consists of seven sections. Section 2 will briefly outline Wada's (2001a) tense theory. Section 3 will first summarize and criticize previous studies, and then clarify the points to be considered. After constructing the temporal structures of PPF- and BGT-sentences within the framework of Wada (2001a) in section 4, I will, in section 5, explain the differences and similarities between the two sentence types (i.e. the points clarified in section 3 ) on the basis of these temporal structures. In section 6, I will draw crucially on the notion of grammaticalization to account for some remaining problems that are not explained in the previous section. Section 7 offers concluding remarks.

1 This paper focuses on exploring why (and how) PPF-sentences are different from and similar to BGT-sentences with respect to tense phenomena, but not on exploring thoroughly factors which cause sentences containing the present progressive to receive future time reference. In the latter are included verb types, temporal adverbials, and the linguistic environment in which present progressive sentences occur.

${ }^{2}$ As an anonymous reviewer has pointed out, SPF-sentences can also represent the near future, assured future, and present-orientation. Because of the lack of space, I cannot make a detailed comparison of SPF-sentences and the two types of sentences dealt with in the main text (i.e. BGT- and PPF-sentences). For a brief description of SPFsentences, see note 17 . 
2. The Theory of Tense in Wada (2001a)

I will start by briefly outlining Wada's (2001a) tense theory, which provides a basic framework for explaining tense phenomena of PPF- and BGTsentences systematically. This tense theory is systematic in that it aims to consider tense phenomena comprehensively in terms of principles, hypotheses, and "characteristic features" that belong not only to the grammatical field of tense but also to other grammatical fields such as verbs (including auxiliaries), modality, aspect, and context. The basic assumption of the analysis based on this theory is that semantic interpretations of tense forms (including $\mathrm{W}$-sentences, BGT-sentences, simple present sentences, and present progressive sentences) arise from their temporal structures under the influence of other semantic, pragmatic, and syntactic factors. ${ }^{3}$ In this section, I will survey some fundamental points of the theory that are necessary for constructing the temporal structures of PPF- and BGT-sentences.

First of all, this theory assumes that in addition to a main verb, an auxiliary verb can express its own situation and thus its own event time (cf. Jannsen (1996), Nakau (1994)). ${ }^{4}$

(1) a. John may come.

b. Nancy is leaving for Europe (tomorrow).

c. Tom is going to play tennis (tomorrow).

Under this assumption, in (1a), the event time of the modal may (the time of a possibility indicated by the speaker which holds at the time of utterance) is expressed in addition to the event time of the lexical verb come. In a similar way, sentence (1b) contains the event time of the progressive auxiliary be as well as the event time of the present participle leaving, and sentence (1c) includes the event time of be going to as well as the event time of the infinitive play. Note that be going to is a series of words which has become a lexically frozen unit through grammaticalization (cf. Hopper and Traugott (1993)), and in the present theory, such a unit as a whole is assumed to represent one event time.

Second, this tense theory regards English finite and non-finite verbs as absolute and relative tense forms, respectively. Absolute tense forms con-

3 The term semantic interpretation is used to represent an interpretation obtained in a specific context, which is differentiated from the general meaning represented by a given form itself.

${ }^{4}$ The term situation is a cover term representing an action, event, state of affairs, etc. 
tain both an $\mathrm{A}$ (bsolute tense)-component and an $\mathrm{R}$ (elative tense)-component, while relative tense forms consist only of the R-component. ${ }^{5}$ The A-component is a tense component whose temporal information is represented by a tense morpheme that changes according to person, number, and mood (i.e. an A-morpheme); the R-component is a tense component whose temporal information is represented by a verb stem and, if any, a tense morpheme that does not change according to person, number, and mood (i.e. an Rmorpheme).

Let me first illustrate English absolute tense forms. With the finite verb plays, for instance, the tense suffix $-s$ is an A-morpheme because it changes according to person, number, and mood, and thus relates to the A-component; the verb stem play, by definition, relates to the R-component. An A-morpheme contributes to constructing a time-sphere, a time length or range established in the grammatical system of a language. English has two A-morphemes, the present and the past tense morphemes represented by $-s$ and $-e d$, respectively. In the default (or normal) case, the choice of absolute tense forms is based on speech time as a pivot. In this case, the present time-sphere, constructed by $-s$, includes speech time, while the past time-sphere, constructed by -ed, lies completely before speech time. A verb stem contributes to representing an event time, namely a time point or interval of the relevant part of the situation talked of. The event time is located somewhere in the time-sphere because the R-component is subordinate to the A-component and the time-sphere is longer than the event time as a time concept.

The temporal structures of the two absolute tense forms (the present and the past tense forms) in English — whose pivotal time is speech time-are diagrammed as follows:

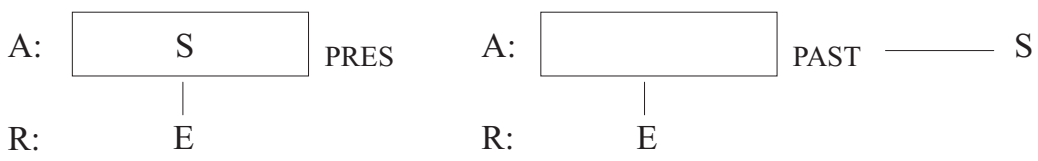
Figure 1: (i) Present Tense Form
(ii) Past Tense Form

$\mathrm{A}$ and $\mathrm{R}$ symbolize the $\mathrm{A}$ - and the R-component, respectively; the rectangle

5 The term absolute in the A-component comes from the fact that this component is connected with grammatical deixis. The term absolute in absolute tense forms comes from the claim that they contain the A-component in their tense structure. 
with subscript PRES represents the present time-sphere, and the rectangle with the subscript PAST the past time-sphere; E stands for event time and $\mathrm{S}$ for speech time; a horizontal line represents temporal subsequence and a vertical line or comma means temporal simultaneity or inclusion.

Let us now consider English relative tense forms. It is assumed in this tense theory that non-finite markers include a component concerning temporal information in their semantics, and thus are a kind of tense morpheme. Since they do not change according to person, number, and mood, they are, by definition, not A-morphemes but R-morphemes, and are thus associated with the R-component. They represent temporal relations between the event time and the time of orientation $(\mathrm{O})$, which is a pivotal time for computing the position of the event time on the time line.

Here I consider the present participle and the bare infinitive, which are directly relevant to this paper. The present participle marker -ing is assumed to represent the temporal relation of simultaneity between the event time and the time of orientation (cf. Declerck (2006), Wierzbicka (1988)). In the sentence Mary is dancing, for example, the event time of the present participle dancing is interpreted as simultaneous with the event time of is as the time of orientation, because the former verb appears in the complement position of the latter verb. The bare infinitive marker-represented by the zero morpheme $\varnothing$ - can indicate all of the three possible temporal relations between the two times, i.e. anteriority, simultaneity, and posteriority, because its temporal information is assumed to be unspecified (cf. Duffley (1992)). Which temporal relation a given bare infinitive indicates depends on other factors such as the verb type, temporal adverbials, and the linguistic environment in which it occurs. For instance, in (1a) above, the nonstative verb come indicates a posterior relationship between its event time and the event time of the finite verb may as the time of orientation; in John may be a genius, the event time of the stative verb be is interpreted as simultaneous with the event time of may. ${ }^{6}$ The following schemata represent the temporal structures of the present participle and the bare infinitive.
R: E , O
$\mathrm{R}: \mathrm{E}-\mathrm{O} ; \mathrm{E}, \mathrm{O} ; \mathrm{O}-\mathrm{E}$

Figure 2: (i) Present Participle Form

(ii) Bare Infinitive Form

Finally, this tense theory utilizes the notion of temporal focus (cf. De-

${ }^{6}$ See Wada (2001a: Ch. 2) for cases of the anterior relationship between the event time and the time of orientation expressed by bare infinitives (cf. also Duffley (1992)). 
clerck $(1991,2006))$. This notion is defined as a speaker's focus fixed on the time point or interval of (part of) a situation to which the speaker is paying special attention. It plays a role not only in capturing differences between similar tense forms in terms of temporal orientation, but also in modeling temporal structures of a given tense form.

Let me illustrate how this notion works by comparing W- and BGT-sentences. Consider:

(2) a. Bruce will leave for Japan.

b. Bruce is going to leave for Japan.

Under the assumption concerning auxiliaries that we saw above, both sentences in (2) contain two event times in their temporal structures: $E_{1}$, i.e. the event time associated with the auxiliary (will or be going to), and $\mathrm{E}_{2}$, i.e. the event time of the infinitive. The properties of non-stativeness of the infinitive leave, together with those of the complement position, make the relationship between $E_{1}$ and $E_{2}$ that of posteriority. $E_{1}$ is located at the same time as speech time because of the presentness indicated by the finite verb. Thus, $E_{2}$ is located in the future. In these respects, the temporal structures of W- and BGT-sentences are the same. However, the position of temporal focus (TF) is different between them in typical cases, as shown in Figure $3 .^{7}$

A:

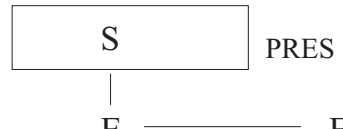

$\mathrm{R}$ :

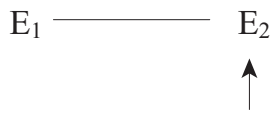

TF

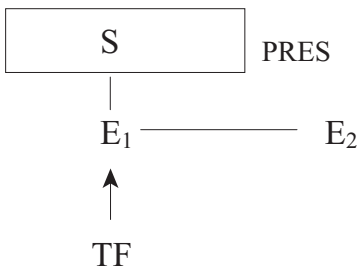

(ii) Be Going To-Sentences

Figure 3: (i) Will-Sentences

This difference models the intuition that basically, W-sentences are futureoriented, while BGT-sentences are present-oriented. Moreover, temporal focus contributes to forming a variety of temporal structures of $\mathrm{W}$ - and

\footnotetext{
7 I regard the non-intentional or predictive use (in a general sense) of W-sentences as their typical use, the only one I will consider in this paper (this use is divided into two types, the predictive and the simple-future use in my sense, which will be defined and discussed in section 6). For the temporal structure of their intentional use, see Wada (2001a: Ch. 7). Typical cases of BGT-sentences are semantic interpretations deriving from the basic temporal structure of BGT-sentences, as we will see in the main text. See also note 13 .
} 
BGT-sentences (cf. Wada (2001a)).

Before closing this section, note that this tense theory is "compositional" in that all the elements constituting a tense form individually contribute to its basic temporal structure. For example, all the elements constituting BGT-sentences as tense form (the finite verb be consisting of a tense morpheme and a verb stem, the present participle going, to, and the infinitive) do not constitute a fused, unpartitioned whole; instead, they all individually contribute to their basic temporal structure.

Now that I have surveyed the basic framework which my analysis is based on, I will, in the next section, briefly review previous studies on comparisons between PPF- and BGT-sentences and summarize the points to be discussed, and then show how the theory of tense outlined in this section provides a basis for constructing the temporal structures of these two sentence types, in terms of which I will explain those points.

\section{Review of Previous Studies and the Points to Be Considered}

I will first survey basic points of Leech (1987, 2004), Swan (1995), and Kashino (1999) as representative previous studies, pointing out problems and insufficiencies common to them. I will then categorize those problems and insufficiencies into three points to be considered in this paper.

\subsection{Previous Studies}

\subsubsection{Leech $(1987,2004)$}

First, I will summarize the findings of Leech $(1987,2004)$. He states that while both BGT- and PPF-sentences refer to "a future happening anticipated in the present," the former express the future outcome of a present intention or cause, but the latter represent a future event expected by means of a present plan, program or arrangement.

Their similarities are illustrated by the fact that the two sentence types not only tend to refer to the near future, but can also refer to the (near) future without future time adverbials, as shown in (3), and by the fact that they can nevertheless refer to the remote future, as in (4).

(3) a. I'm taking Mary out for a meal (tomorrow).

b. I'm going to take Mary out for a meal (tomorrow).

(4) a. When I grow up, I'm joining the police force.

b. I'm going to do what I like when I retire.

One difference between BGT- and PPF-sentences is that the former tend to represent an intention, as illustrated by $(5 \mathrm{~b})$, but the latter tend to express 
an arrangement, as exemplified by (5a):

(5) a. I'm taking Mary out for dinner this evening.

b. I'm going to take Mary out for dinner this evening.

This tendency of PPF-sentences explains the lower acceptability of (6a). We usually regard someone's watching TV alone as an event based on his/her intention, but not as an arrangement or something socially predetermined. (6b) is perfectly acceptable because the tendency of BGT-sentences in question matches with the properties of the event in question.

(6) a. ?I'm watching TV this evening.

b. I'm going to watch TV this evening.

Another difference is that PPF-sentences are possible only with dynamic verbs involving human agency (cf. Wekker (1976)), whereas BGT-sentences do not have such a restriction. Thus we see the difference in acceptability in (7):

(7) a. \{John's getting up/*The sun is rising \} at 5 o'clock tomorrow.

b. \{John's going to get up/The sun is going to rise $\}$ at 5 o'clock tomorrow.

\subsubsection{Swan (1995)}

Next, I will briefly outline Swan (1995). In some respects, he makes similar statements to Leech. For example, with respect to the similarities between BGT- and PPF-sentences, he states that they both refer to "actions and events that have some present reality." With respect to their differences, he points out, for instance, that PPF-sentences are required to contain dynamic verbs involving human agency, but BGT-sentences are not.

With regard to other differences between the two sentence types, however, he not only makes a slightly different statement from Leech's, but also provides a more detailed description. For example, PPF-sentences express personal or fixed arrangements and fixed plans, and they are often used with verbs of movement. On the other hand, although BGT-sentences can be used to refer to plans (usually informally), they are mainly used either to emphasize an intention or previous decision, or to predict a future action or event for which there is present evidence. These statements are illustrated by (8) and (9):

(8) a. Are you doing anything this weekend? (asking about arrangement-more natural than Are you going to do anything this weekend?)

b. Are you going to do anything about that letter from the tax 
people? (pressing to know what has been decided-more natural than Are you doing anything about that letter ...?)

(9) a. I'm getting a new job. (It's already arranged.)

b. I'm going to get a new job. (I've decided to.)

As another difference of the two sentence types, he points out that PPFsentences, unlike BGT-sentences, usually cannot be used to talk about permanent states, as shown in (10):

(10) a. Their new house is going to look over the river.

b. *Their new house is looking over the river.

He further notes that although both BGT- and PPF-sentences can be used to express refusals, the latter usually express emphatic ones. They are exemplified by (11) and (12):

(11) You're not \{playing/going to play\} football in my garden.

(12) I'm sorry, you're not taking my car.

\subsubsection{Kashino (1999)}

Kashino (1999), like Leech, states that PPF-sentences express a future event anticipated by virtue of a present plan, program or arrangement. With regard to BGT-sentences, he clearly distinguishes between the intentional use, which indicates that the subject's intention holds at present, and the predictive use, which shows that the speaker's prediction about a future event is based on present evidence or some indication. Using this distinction, he states that BGT-sentences of the intentional use are compatible only with self-controllable verbs or verbs with the subject's intention, but those of the predictive use do not have such a restriction. Therefore, when BGT-sentences describe events without the subject's intention, they are seen as conveying the speaker's prediction, as illustrated by (13).

(13) a. He's going to die next week.

b. The tickets are going to go on sale next week.

Because PPF-sentences do not have the predictive use, the sentences containing verbs without the subject's intention in (14) cannot be interpreted as expressing the speaker's prediction; they are taken as conveying a plan or arrangement previously determined.

(14) a. He's dying next week.

b. The tickets are going on sale next week.

The failure of PPF-sentences to have the predictive use is illustrated by the fact that unlike BGT-sentences, PPF-sentences usually cannot co-occur with "futurate open conditionals" expressing conditions which may or may not be fulfilled in the future (cf. Declerck (2006: 103)), with which W-sen- 
tences of the predictive use can co-occur, as in (15).

a. *You're breaking that chair if you're not careful.

b. You are going to break that chair if you're not careful.

c. You will break that chair if you're not careful.

Although Kashino himself does not mention it explicitly, I will briefly state here why W-sentences with futurate open conditionals show a predictive reading, which will be relevant in section 6.2.2. As stated in Dancygier (1998: 47), an additional predictive expression in a W-sentence with a futurate open conditional makes the whole sentence unacceptable, because such an addition causes the redundancy of prediction in that W-sentence, as exemplified by *If it rains, the match will be canceled, and I predict it will (here, and I predict is an additional predictive expression). This suggests that will in $\mathrm{W}$-sentences with futurate open conditionals like the one in (15c) indicates prediction.

Kashino further points out other cases where PPF-sentences receive different interpretations from BGT-sentences. In (16) below, for example, the PPF-sentence indicates that the situation is already arranged and the object's referent also knows it, whereas the BGT-sentence implies that the speaker alone knows it (cf. Thomson and Martinet (1986)).

(16) a. I'm meeting Tom at the station at six.

b. I'm going to meet Tom at the station at six.

Another difference between the two sentence types is that when giving an excuse for refusing an invitation, the speaker normally chooses PPF-sentences, not BGT-sentences, to emphasize the existence of a promise previously made (cf. Leech $(1987,2004))$. This is exemplified by (17):

A: Can you come and see us this evening?

B: I can't. $\quad$ I'm playing/I'm going to play\} squash with Sam.

3.2. Problems and Insufficiencies in These Previous Studies

Now, let me point out problems and insufficiencies in these previous studies. First of all, although they state that PPF-sentences are preferable to BGT-sentences in certain situations, and vice versa in others, they do not provide a fundamental explanation for why things are the way they are (e.g. (8), (11) and (17)).

Next, although both PPF- and BGT-sentences are considered to represent future situations that have some present reality (e.g. (9)) or to refer to the near future (e.g. (3) and (5)), the reason for this is not clarified. It also remains unexplained why the two sentence types do not necessarily refer to the near future, as in (4) above. 
Furthermore, the previous studies disagree on some points with respect to the semantic interpretations of PPF- and BGT-sentences. For instance, with regard to the notion of arrangement represented by PPF-sentences, Leech $(1987,2004)$ regards it as something "socially predetermined in the past," while Swan (1995) takes it as a "personal arrangement," and therefore, their views on this notion seem to be slightly different. Moreover, although the notions of intention and (previous) decision are associated with BGT-sentences in these previous studies, some other studies (e.g. Nesselhauf (2007), Hirtle and Curat (1986)) state that PPF-sentences can be used to express an intention, and still others (e.g. Thomson and Martinet (1986)) indicate that PPF-sentences can represent a decision, especially when used with verbs of movement (cf. also Hirtle and Curat (1986)). ${ }^{8}$ In addition, Leech (2004: 62) notes that the difference in semantic interpretation between the arrangement expressed by PPF-sentences and the intention expressed by BGT-sentences is slight.

Further issues that need to be addressed are (i) why PPF-sentences tend not to be compatible with stative verbs, but BGT-sentences are, as shown in (10) above; and (ii) why BGT-sentences have the predictive use, while PPFsentences do not, as illustrated by (15).

\subsection{Issues To Be Discussed}

The problems and insufficiencies discussed above can be summarized as follows:

(18) a. Why is it that PPF-sentences express arrangements and plans as their main semantic interpretations, while BGT-sentences express intentions and previous decisions as their main semantic interpretations?

b. Why is it that PPF-sentences and BGT-sentences have a common semantic range (e.g. a tendency to refer to the near future) and that each encroaches on the main semantic interpretations of the other (e.g. some PPF-sentences express intentions and previous decisions that are considered to be the main semantic interpretations of BGT-sentences)?

c. Why is it that in comparison with BGT-sentences, PPF-sen-

${ }^{8}$ Although it has often been pointed out in previous studies such as Swan (1995) and Kashino (1999) that PPF-sentences tend to be used with verbs of movement, this is not a strong tendency, as is evident from the data in the main text (cf. Wekker (1976: 111)). 
tences refer to a narrower semantic range or are more restricted in use?

In order to provide a fundamental explanation for these points systematically and from a unified point of view, I will, in the next section, construct the temporal structures of PPF- and BGT-sentences in the theory of tense outlined in section 2, and then show in section 5 that the differences and similarities between the two sentence types (which are summarized in the three points in (18)) all arise from their temporal structures.

\section{The Temporal Structures of PPF-sentences and BGT-sentences}

\subsection{Constructing Temporal Structures}

In constructing the temporal structures of PPF- and BGT-sentences, I will follow work on grammaticalization to take in both the view that a form associated with a function (or meaning) acquires a new function (or meaning) only gradually and in a motivated way in the course of grammaticalization, and the view that not only a newly acquired function (or meaning) but also a former function (or meaning) can be retained in that form synchronically (cf. Hopper and Traugott (1993), Traugott and Dasher (2002), Traugott and Heine (1991)). It is stated in these studies that the grammaticalization of BGT-sentences has started from the stage of the progressive of go with a purposive clause; then, by reanalysis they have come to be viewed as the "future" auxiliary with a verb of activity, and finally reached the stage of expressing the "future" tense. (Note, however, that I regard BGT-sentences as a future time expression, but not as a future tense form, as we will see below.) In present-day English, the latter two stages are retained in BGTsentences as tense form. Combining this theory of grammaticalization with the tense theory of Wada (2001a) enables me to account for a variety of tense phenomena of PPF- and BGT-sentences fundamentally and in a motivated way.

In this section, I will restrict myself to the basic stage (the "future" auxiliary with a verb of activity) of BGT-sentences and postpone a consideration of the stages following the basic one in the process of grammaticalization until section 6. By doing so, I can potentially account for why some native speakers of English make a sharp distinction between BGT- and W-sentences, whereas others do not (see Wada (2001a: Ch. 7)). I will argue that in contrast to BGT-sentences, PPF-sentences have only a single stage with future time reference, which derives from progressive sentences by virtue 
of a change in their temporal structure. ${ }^{9}$ The reason why BGT-sentences have more than one temporal structure with future time reference, but PPFsentences have only the single one, will be explored in section 6 .

\subsection{PPF-sentences}

I will start with the temporal structure of PPF-sentences. Because the finite verb be is a present tense form, its temporal structure is the one shown in Figure 1 (i) above. On the other hand, the non-finite verb, i.e. the present participle, has the tense structure of simultaneity shown in Figure 2 (i) above. The properties of the complement position cause the event time of the finite verb (i.e. $E_{1}$ ) to be the time of orientation for the event time of the present participle (i.e. $\mathrm{E}_{2}$ ). These observations enable me to present the basic temporal structure of present progressive sentences in Figure 4 (i). This temporal structure changes into the temporal structure shown in Figure 4 (iii) through the one shown in Figure 4 (ii) when present progressive sentences are interpreted as referring to the future. ${ }^{10}$

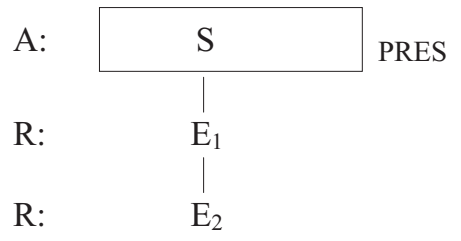

Figure 4: (i) Present Progressive Stage 1
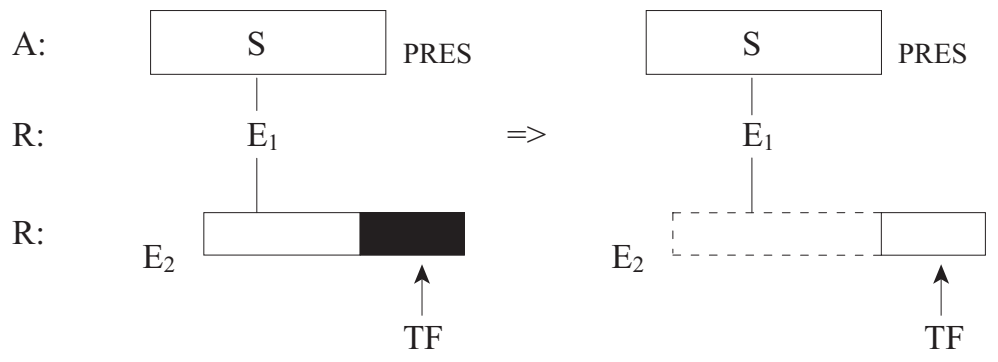

Figure 4: (ii) Present Progressive Stage 2 (iii) Present Progressive Stage 3

9 Nesselhauf (2007) observes that PPF-sentences started to be prevalent in late ModE. This suggests that PPF-sentences have derived through grammaticalization from progressive sentences, which are said to have become more common in early ModE.

10 The claim that the temporal structure in Figure 4 (i) is basic and the one in Figure 4 (iii) is derivative is also supported by the fact that present progressive sentences representing progressive aspect are much less restricted in use than PPF-sentences. 
The mechanism for this change is as follows. Let me first explain why and how the temporal structure in Figure 4 (ii) emerges. Smith (1981: 374-375) states that the present progressive form, which represents imperfective aspect or a simple process, is reinterpreted as representing an extended process consisting of the preliminary stage of a situation and its outcome in contexts with future time reference (cf. also Hirtle and Curat (1986) and Prince (1982)). I will introduce this notion of extended process into the temporal structure of present progressive sentences, connecting the extended process as a whole with the present participle because the progressive be does not have substantial semantic content and thus merely represents a very schematic situation. (This is in keeping with the Cognitive Linguistic view that the progressive be merely serves to connect the present-participle situation to the time line.) As a result, the temporal structure in Figure 4 (ii) emerges, where the white rectangle related to $\mathrm{E}_{2}$ symbolizes the preliminary stage of the present-participle situation and the black rectangle related to $\mathrm{E}_{2}$ stands for the outcome stage of that situation; at this stage, this type of sentence is reconsidered to represent the present-participle situation as perfective or heterogeneous in Smith's (1981) terms, the end of the preliminary stage leading immediately to the beginning of the present-participle situation itself.

Note that the temporal focus is directed not at $E_{1}$ but at $E_{2}$. It is hard for $E_{1}$ to receive the temporal focus in that the semantic content of the progressive be is impoverished or schematic, and at the same time, the preliminary stage of the present-participle situation, whose semantic content is rich enough, shares the same time range as the schematic situation of the progressive be. The reason why the temporal focus is directed only at the outcome stage (part of the target situation) is as follows: in order for PPF-sentences to indicate the very high degree of future actualization of the situation, it is more natural to put our focus on the outcome stage, but not on the preliminary stage, of that situation in that the former case directly reflects the speaker's emphasis on the occurrence of the situation itself.

Once the outcome stage receives the temporal focus, the other part, i.e. the preliminary stage, becomes a background in the temporal structure. As a result of this process, $\mathrm{E}_{2}$ is reinterpreted as corresponding only to the time of the outcome stage and thus as coming temporally after $E_{1}$, the event time of the schematic situation represented by the progressive be, which functions as the time of orientation for $E_{2}$ for the reason mentioned above. Thus, the temporal structure of PPF-sentences in Figure 4 (iii) emerges. This is similar to the temporal structure of BGT-sentences in Figure 3 (ii) in that 
in both cases $E_{1}$ obtains in the present and $E_{2}$ in the future. However, what should be stressed is that the preliminary stage of PPF-sentences is the backgrounded part of the present-participle situation associated with $\mathrm{E}_{2}$, and its existence is indirectly implied by the progressive be, whose schematic situation shares the same time range as the preliminary stage. This is not the case with BGT-sentences, as we will see in the next subsection.

Now, let me justify my claim that PPF-sentences consist of two different stages of a situation corresponding to two different times. The first argument for this claim is that PPF-sentences can co-occur with adverbials referring to two different times.

(19) a. Now I'm flying down there tomorrow. (Webb (1968: 155))

b. Right now she's leaving tomorrow and that's that.

(boards.babycenter.com/bcus1525975/messages/4754/16) In (19a), for example, now specifies $\mathrm{E}_{1}$, the time of the schematic situation represented by be, which indirectly implies the existence of the preliminary stage of the present-participle situation, and tomorrow specifies $\mathrm{E}_{2}$, the time of its outcome stage.

Second, a sentence like (20) can be presented as evidence for my claim.

(20) Jim's probably leaving tomorrow.

(Declerck (2006: 187)) Declerck (2006: 187-188) observes that in (20) the likelihood represented by probably can concern either the existence of a present arrangement/intention (corresponding to the preliminary stage of the present-participle situation) or the future occurrence of the present-participle situation itself (i.e. the outcome stage of that situation). This observation suggests that PPF-sentences refer to two different stages corresponding to two different times.

\subsection{BGT-sentences}

Let me now move to the basic temporal structure of BGT-sentences. Although the temporal structure of BGT-sentences has already been shown in Figure 3 (ii), that is merely a simplified version of the basic stage. To make a full comparison with the temporal structure of PPF-sentences in Figure 4 (iii), we need a detailed version of it. It is assumed in the present theory that the situation represented by the lexically frozen unit be going to has an internal structure which is divided into subparts. Under this assumption, we can present the basic temporal structure of BGT-sentences in Figure 5. 


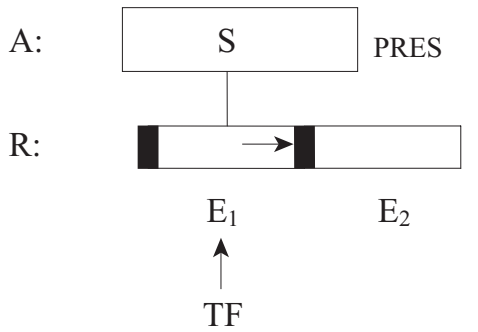

Figure 5: Be Going To-Sentences (Detailed Version)

This assumption is justified by the view that the internal structure of be going to originally reflects the semantic properties of the progressive form of $g o$ and the preposition to, and partially preserves them even now. In the course of grammaticalization, the meaning of be going has changed by metaphorical extension from a spatial meaning, i.e. the speaker being on the way from one place to another, to a more subjective or temporal meaning, i.e. the speaker or the situation surrounding him/her being metaphorically at a point of the time line extending from a time point in the past to a time point in the future (cf. Sweetser (1988)). The meaning of to has also changed by metaphorical extension from a spatial path to a temporal path in the speaker's mind, because the purpose-clause time (the time of the to-infinitive) comes after the main-clause time (the time of be going) (cf. Duffley (1992)).

These semantic properties are reflected in the internal structure of the situation represented by be going to, which has arisen from the reanalysis of be going and a purpose clause. The be going part indicates that the speaker or the target situation is subjectively moving along the time line toward the future and is now at the time of orientation (speech time in the case of BGT-sentences in the present tense form), which entails that either $\mathrm{s} /$ he has already departed from the starting point or the target situation surrounding him/her has already started to occur. Thus, the internal structure of the be going part necessarily contains the starting point or onset of the subjective movement on the time line (corresponding to the leftmost black part of the rectangle connected to $E_{1}$ in Figure 5) as well as the process of subjective movement toward the future occurrence of the infinitive's situation (corresponding to the white part of that rectangle). The temporal path represented by the to which is attached to be going includes not only the process itself but also the end of it, because a path by definition reaches the goal. The arrow in the rectangle associated with $\mathrm{E}_{1}$ in Figure 5 symbolizes this temporal path, and the right-hand black part of this rectangle represents 
its end point or coda.

Now that we have seen the internal structure of the be going to part, let us next consider how the infinitive part contributes to the basic temporal structure of BGT-sentences. As we saw in Figure 2 (ii), the bare infinitive can tense-structurally indicate the three possible temporal relations between the event time and the time of orientation. However, the semantic properties of to discussed above require the infinitive's situation to come temporally after the situation represented by be going to. Thus, $\mathrm{E}_{2}$, associated with the infinitive, is necessarily posterior to $\mathrm{E}_{1}$, associated with be going to.

With BGT-sentences, the be going to part corresponds to the preliminary stage in that, as we have seen, its internal structure represents the subjective movement on the time line toward the future occurrence of the target situation; the situation itself, the outcome stage of it, is represented by the infinitive's part. What should be stressed here is that in contrast to the case of PPF-sentences, the preliminary stage and the outcome stage of BGTsentences are represented by two different verbal units related to two different event times: the preliminary stage is associated with $E_{1}$ and the outcome stage with $\mathrm{E}_{2}$.

Here I will justify my claim that BGT-sentences consist of two different stages corresponding to two different times. A first argument is again the co-occurrence of BGT-sentences with adverbials referring to two different times. Consider (21):

(21) a. Now we are going to have no money at the end of the month.

(Haegeman (1989: 297))

b. Now I'm going to wake up tomorrow.

(query.nytimes.com/gst/fullpage.html?res=9D

02E0DF1F3AF932A35757C0A961958260)

Further support for my claim emerges from the fact that since BGTsentences tense-structurally require the infinitive's situation to be posterior to the situation represented by be going to, they necessarily refer to two different times, i.e. the present and the future in the case of BGT-sentences in the present tense form. The statement that the infinitive's situation necessarily obtains in the future is verified by the fact that even if it is specified by now, it is interpreted as existing in the future, not in the present, as in (22), where the position of now normally requires the time adverb to specify the infinitive's situation.

(22) They are going to wash the dishes \{now/later\}.

This sharply contrasts with the fact that with PPF-sentences, the presentparticiple situation when specified by now exists in the present, as in (23) 
(cf. Kashino (1999)).

(23) They are washing the dishes $\{$ now/later\}.

\section{Explanation}

5.1. Similarities between PPF-sentences and BGT-sentences

Now I will explain the three points summarized in section 3.3 with the temporal structures of PPF- and BGT-sentences shown in Figure 4 (iii) and Figure 5 above. I will start with the first half of the second point in (18b), the reason why PPF- and BGT-sentences have a common semantic range. This is a natural consequence of the common range of the temporal structures of the two sentence types: they both contain the preliminary stage (which holds in the present) and the outcome stage (which occurs as a result of the occurrence or existence of the preliminary stage).

With this in mind, let me first explain the similarity between PPF- and BGT-sentences pointed out by Leech $(1987,2004)$ and Swan (1995), namely that they represent future situations that have some present reality. This comes from the common range of their temporal structures in which the preliminary stage obtains in the present.

By the same token, I can also explain both why the two sentence types tend to refer to the near future without adverbials representing specific times, and why they can refer to the remote future. (3) and (4) are repeated here as (24) and (25).

(24) a. I'm taking Mary out for a meal (tomorrow).

b. I'm going to take Mary out for a meal (tomorrow).

a. When I grow up, I'm joining the police force.

b. I'm going to do what I like when I retire.

In (24a), the existence of the progressive be indirectly implies the preliminary stage leading up to the outcome of the present-participle situation; in (24b), the existence of the lexically frozen unit be going to directly indicates the preliminary stage. Actions and events whose preliminary stage has already occurred tend to occur soon thereafter. Hence, both PPF- and BGT-sentences tend to refer to the near future. However, this nearness in time is merely a pragmatic implicature, because the preliminary stage can continue for a long time. Therefore, as shown in (25), both PPF- and BGT-sentences can refer to the remote future, especially by virtue of the presence of a time adverbial or context indicating it.

With respect to the second half of the second point in (18b), namely the reason why one of the two sentence types encroaches on the main semantic 
interpretations of the other type, I will return to it at the end of the next subsection.

\subsection{Arrangement/Plan vs. Intention/Decision}

Let me move to the first point in (18a), namely the reason why PPFsentences express arrangements and plans as their main semantic interpretations, while BGT-sentences express intentions and previous decisions as their main semantic interpretations. This comes from the differences of temporal structures of the two sentence types. Although the preliminary stage is included in the temporal structures of both types, it is directly indicated by be going to with BGT-sentences, while it is indirectly implied by be with PPFsentences. More specifically, in the case of PPF-sentences, the preliminary stage is a backgrounded part of the present-participle situation and combines with the outcome stage to constitute one single verbal unit, and that is implied by the existence of the progressive be (an explicit element), whose schematic situation shares the same time range as that preliminary stage. In the case of BGT-sentences, however, the preliminary stage is represented by the lexically frozen unit be going to, a verbal unit different from the infinitive expressing the outcome stage.

This difference plays a vital role. Because the preliminary stage and the outcome stage of PPF-sentences are connected with one verb (the present participle), the degree of coherence between them is high. Moreover, since the preliminary stage is regarded as backgrounded, its existence is presupposed and thus is not the object of assertion, which suggests that the preliminary stage is hard to halt or cancel. Therefore, it is highly likely with PPF-sentences that, once the preliminary stage is underway, the outcome stage is regarded as certain to occur.

In contrast, because the preliminary stage and the outcome stage of BGTsentences are connected with two different verbal units (be going to and the infinitive), the coherence of them is lower than in the case of PPF-sentences, where both stages are connected with one verbal unit. This line of reasoning is supported by a general cognitive pattern in which two related entities in different positions (or attached to different objects) tend to work together less closely than the same two related entities in a single position (or attached to a single object). Moreover, the preliminary stage represented by be going to is not backgrounded and thus not presupposed in that it is 
represented by an explicit element. ${ }^{11}$ This lower degree of coherence in BGT-sentences, together with the failure of the preliminary stage to be backgrounded, makes it possible for the speaker to more easily halt or cancel the preliminary stage (immediately) after its occurrence, which in turn implies a lesser degree of necessity of the occurrence of the outcome stage. On the other hand, the occurrence of the preliminary stage strongly suggests the occurrence of the outcome stage unless otherwise indicated, because the outcome stage occurs as a natural result of the occurrence or existence of the preliminary stage which receives special focus, i.e. temporal focus. From these observations, I conclude that in the case of BGT-sentences, the outcome stage is also regarded as certain to occur, but the degree of certainty is not as high as in the case of PPF-sentences.

Let me now compare the notions of arrangement and (fixed) plan with the notions of intention and previous decision. Considering the definitions of these notions common to several dictionaries (e.g. Crowther et al. (1995) and Sinclair et al. (2006)), we can define them as follows: an arrangement is defined not only as a plan or preparation but also as an agreement with someone to do something; a plan is an idea thought about in detail in advance or something that one has decided to achieve; an intention is an aim or something that one proposes or plans to do; and, a decision is a conclusion reached or a judgment. Indeed it is difficult to distinguish completely between these notions, but we can at least say that the former two notions (arrangement and plan) tend to make the occurrence of the situation more certain than the latter two, intention and previous decision. An agreement with someone and something thought about in detail beforehand are generally more difficult to halt or cancel than a mere aim or conclusion in that the former two notions - which normally cannot be canceled unilaterally or have been considered carefully-require more effort for interruption or cancellation than the latter two notions, which can be handled simply or without consulting others. This suggests that situations based on the notions of

11 The reason why the preliminary stage of BGT-sentences is not presupposed is attributed partly to the presence of variants of their temporal structures (as we will see in section 6.1), while the reason why the preliminary stage of PPF-sentences is presupposed is attributed partially to the presence of a single temporal structure. It is usually the case that if more than one type of situation is possible with the presupposed or omitted part, it is hard to decide on one without a detailed context. Therefore, we can claim that the preliminary stage of BGT-sentences, i.e. one possible situation represented by be going to, is not presupposed. 
arrangement and plan are more certain to occur than those based on the notions of intention and previous decision.

From the discussion thus far, I conclude that the notions of arrangement and plan match better with the temporal structure of PPF-sentences, while the notions of intention and previous decision align more closely with the temporal structure of BGT-sentences. This is one answer to the point in (18a).

Let me briefly repeat my explanation for these differences between the semantic interpretations of PPF- and BGT-sentences by using (5), (8) and (9), repeated here as (26), (27) and (28):

(26) a. I'm taking Mary out for dinner this evening.

b. I'm going to take Mary out for dinner this evening.

a. Are you doing anything this weekend? (asking about arrangement-more natural than Are you going to do anything this weekend?)

b. Are you going to do anything about that letter from the tax people? (pressing to know what has been decided-more natural than Are you doing anything about that letter...?)

(28) a. I'm getting a new job. (It's already arranged.)

b. I'm going to get a new job. (I've decided to.)

In (26), the differences of the temporal structures of the two sentence types discussed above trigger an arrangement reading for the PPF-sentence and an intention reading for the BGT-sentence. The other pairs can be explained in the same way.

As to the notion of arrangement, it should be considered here that Leech regards it as something socially predetermined while Swan regards it as personal. An anonymous reviewer has pointed out that this discrepancy is apparent, and both types of arrangements are interpersonal, implying that only the level of arrangement is different (i.e. public vs. private matters). Therefore, personal as well as socially-determined arrangements require more effort for interruption or cancellation than intentions or decisions; the former match the temporal structure of PPF-sentences better than that of BGT-sentences.

It should also be noted here that in my temporal structure-based analysis, main semantic interpretations are prototypical ones arising from each temporal structure; moreover, not only are non-typical semantic interpretations associated with a temporal structure possible as far as they are motivated in some way by that temporal structure, but they can also be prototypical ones arising from another temporal structure on a scale of the same notion. This 
enables me to explain why PPF-sentences can represent the main semantic interpretations of BGT-sentences, such as intentions and decisions. Intentions/decisions and arrangements/plans can both be put on the continuum of certainty of occurrence. When intentions and decisions are strong, their meanings become closer to arrangements and plans as far as the degree of certainty of their occurrence is concerned, because strong intentions and decisions are more difficult to halt or cancel than simple ones. Therefore, the temporal structure of PPF-sentences can match with strong intentions and decisions because the latter are still motivated by the former.

Even if intentions and decisions are not so strong, PPF-sentences can convey these notions when the higher degree of certainty of the occurrence of the target situation is indicated by other means. Consider the following:

(29) I'm going home tonight.

Because of the first-person subject and the presence of the time adverb specifying the near future, sentence (29), even if interpreted as expressing a simple intention or decision, shows a higher degree of certainty of the occurrence of its situation. These notions are still motivated by the temporal structure of PPF-sentences. Hence the use of a PPF-sentence.

This line of explanation further accounts for why PPF-sentences which are difficult to view as indicating arrangements or plans are sometimes considered to be odd, as shown in (6), repeated here as (30).

a. ?I'm watching TV this evening.

b. I'm going to watch TV this evening.

The reason for the oddness of (30a) was that PPF-sentences tend to express arrangements or plans by virtue of their temporal-structural properties, whereas the event of someone watching TV alone on a particular evening is somewhat difficult to view as something arranged. The reason why the notion of arrangement tends to be preserved here is that arrangements and plans are the prototypical interpretations of PPF-sentences and one tends to come up with prototypes. As we saw above, however, the range of semantic interpretations of PPF-sentences can extend from their main (or prototypical) interpretations. I assume that the possibility of such an extension depends on the semantic properties of the target situation or on the individual's judgment if the notion of intention or decision can be involved. Under this assumption, the person who judges sentence (30a) to be less acceptable sticks to the main semantic interpretations of PPF-sentences more strictly than the person who regards sentence (29) as acceptable, although both sentences can convey the subject's intention.

This explanation also explains the acceptability of (14a), repeated as (31). 
(31) He's dying next week.

Because of the third-person subject, this sentence is highly unlikely to express the subject's own intention. Moreover, someone's dying (except for committing suicide) cannot be decided or intended by him/herself. Therefore, this PPF-sentence usually expresses one of its main semantic interpretations, i.e. something socially arranged or the speaker's (personal) arrangement if he or she has the authority to execute a death sentence. The extension in question is difficult with the situation in (31).

The same line of explanation applies to cases where BGT-sentences encroach on the main semantic interpretations of PPF-sentences. The temporal-structural properties of the two sentence types discussed above allow their respective semantic interpretations to be both overlapping and distinct.

\subsection{Other Differences between PPF-sentences and BGT-sentences}

Finally, I will account for the point in (18c), the reason why PPF-sentences refer to a narrower semantic range or are more restricted in use than BGT-sentences. Let me first explain why the constraint as to conscious human agency or human controllability is, in principle, imposed on the use of PPF-sentences. Recall that because of the properties of the temporal structure of PPF-sentences, it is highly likely that the outcome stage will occur. To meet this high degree of certainty, the target situation should be humanly controllable. Only in that case can we decide to make it happen or say with confidence that such a situation will surely follow from our decision. From these observations, I argue that the notion of human controllability matches the temporal structure of PPF-sentences. In contrast, the properties of the temporal structure of BGT-sentences indicate that the degree of certainty as to the future occurrence of the situation is not as high as in the case of PPF-sentences. This allows events outside human control to occur in BGT-sentences because we cannot say with full confidence that such events will surely happen, on one hand, and the temporal structure of BGT-sentences shows a higher possibility that the target situation will not happen, on the other. Thus, the constraint in question is not imposed on the use of BGT-sentences.

What should be stressed here is that the human-controllability constraint is motivated by and derives from the temporal structure of PPF-sentences, but is not inherent to them. If the latter were the case, we could not explain why some speakers judge sentence (32a) to be unacceptable while others judge sentence (32b) to be acceptable, even though both sentences represent something that humans cannot control. 
a. *The sun is rising at 5 o'clock tomorrow. (cf. (7a))

b. The sun is rising at 6:31 tomorrow.

(Prince (1982: 458))

In my explanation, the human-controllability constraint is a strong implicature deriving from the temporal structure of PPF-sentences and is cancelable depending on the context or the speaker. Sentence (32b) is acceptable only when the speaker's certainty of future occurrence of the situation is taken into consideration; the constraint in question is not operative in the mind of those who accept this type of sentence.

My explanation also accounts for why PPF-sentences imply that both the speaker and the object's referent know the arrangement involved, whereas BGT-sentences suggest that only the speaker knows it, as exemplified in (16) above, repeated here as (33):

(33) a. I'm meeting Tom at the station at six.

b. I'm going to meet Tom at the station at six.

It requires more work to halt or cancel an agreement with others than a unilateral decision. For this reason, an agreement with others shows a higher degree of certainty of the occurrence of the target situation than a personal decision; the former is a better match for the temporal structure of PPFsentences, and the latter for the temporal structure of BGT-sentences.

My temporal structure-based explanation further accounts for the differences of pragmatic nuances of the two sentence types shown in section 3. Let us first consider why PPF-sentences express emphatic refusals. Consider (11) and (12) again, repeated here as (34) and (35):

(34) You're not \{playing/going to play\} football in my garden.

(35) I'm sorry, you're not taking my car.

Refusing the preliminary stage of these PPF-sentences, which indicate a higher degree of certainty of future occurrence of the situation, makes the speaker's refusal emphatic because the addressee regards the situation as more certain to occur if the speaker does not refuse it.

From a similar point of view, I can also explain why PPF-sentences, rather than BGT-sentences, are preferred to emphasize the existence of a prior engagement when we refuse an invitation. Let us look at (17) again, repeated here as (36).

(36) A: Can you come and see us this evening?

B: I can't. \{I'm playing/I'm going to play\} squash with Sam.

As we have seen, the temporal structure of BGT-sentences implies a lower degree of certainty of the occurrence of a future situation than that of PPFsentences. Therefore, the use of a BGT-sentence here may strike A as impolite in that it suggests that B does not have a firm appointment and 
could accept his/her invitation if $\mathrm{s} / \mathrm{he}$ wanted to. By contrast, the use of a PPF-sentence may strike A as less impolite because it suggests that it would be difficult or impossible for $\mathrm{B}$ to cancel the prior engagement. Hence the PPF-sentence is preferred here.

\section{Remaining Problems}

This section accounts for the two problems which remain unexplained, namely the compatibility of PPF- and BGT-sentences with stative verbs expressing permanent states and the difference between PPF- and BGTsentences with respect to the possibility of the semantic interpretation of prediction. For these purposes, we first have to see that BGT-sentences can express the temporal structures of the following stages in the process of grammaticalization, but PPF-sentences do not have variants, as I noted above.

6.1. Grammaticalization and the Change of Internal Structure of the Preliminary Stage

Let me start with BGT-sentences. As I mentioned, their temporal structure shown in Figure 5 above is the basic one. Following Wada (2001a: Ch. 7), which is based on standard views of the process of grammaticalization of be going to like the ones noted in section 4.1, I assume that this basic structure changes into the temporal structure in Figure 6 (ii) via the one in Figure 6 (i) in certain contexts. ${ }^{12}$ This means that in present-day English, BGT-sentences have one of these three structures depending on the context. (This does not imply, however, that these three represent an exhaustive list of variants of temporal structure of BGT-sentences.) It should be noted here again that the two temporal structures of BGT-sentences in Figure 6 are also associated with their main (or prototypical) semantic interpretations. In what follows, the stage in Figure 5 is referred to as

12 This approach enables me to argue that for native speakers who make a sharp distinction between BGT- and W-sentences, BGT-sentences do not have the temporal structure in Figure 6 (ii). For them, BGT-sentences cannot contain stative verbs, as shown in (i):

(i) *I wonder if she's going to know you.

(Leech (1987: 59))

In the temporal structures shown in Figures 5 and 6 (i), because the internal structure of the be going to situation contains the coda, the infinitive situation must be nonstative. For that reason, sentence (i) is deemed unacceptable for those native speakers. See also note 15 . 
Stage A, that in Figure 6 (i) as Stage B, and that in Figure 6 (ii) as Stage C. (Note that Stage C corresponds to the stage of "future" tense stated in section 4.1.) As occurs in the case of Stage A, both Stage B and Stage C temporal-structurally change gradually from and are motivated by their own earlier stages. This semantic change is triggered by analogy and/or subjectification.

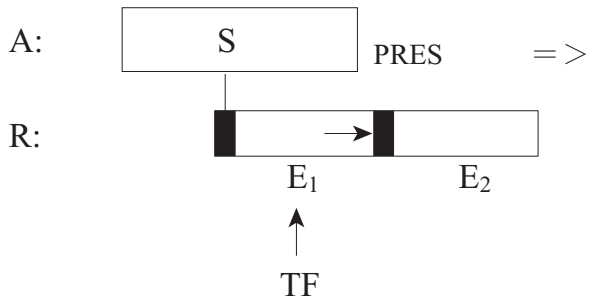

Figure 6: (i) Be Going To-Sentences (Stage B)

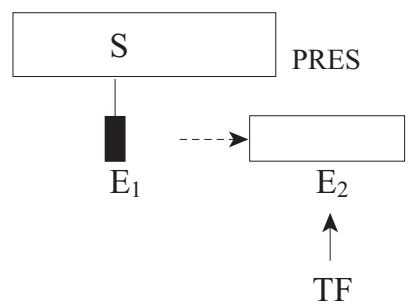

(ii) Be Going To-Sentences (Stage C)

Let me illustrate the mechanism of this temporal-structure change step by step. First, at the beginning of further grammaticalization from Stage A, the onset part of the be going to situation (the preliminary stage), associated with $E_{1}$, is subjectively rather than objectively reinterpreted as obtaining at or around speech time in certain contexts. As a result, BGT-sentences of this type come to have the temporal structure of Stage B. (Unlike in Stage $\mathrm{B}$, the process, but not the onset, of the be going to situation obtains at speech time in Stage A.) Consider (37):

(37) a. "Good," Michael said. "Just sit tight. And I guess that's what I want to say to all of you. Just sit tight. Don't react to any provocation. Give me a few weeks to straighten things out, to see which way the wind is going to blow. Then I'll make the best deal I can for everybody here. Then we'll have a final meeting and make some final decisions.

(Puzo (1969: 411-412))

b. "Wrong!" Buford roared. "It's yours! So from now on you better look behind you when you walk, 'cause one day you're going to get a bullet in your back....

(Gardner (1990: 94))

Take (37a) for example. It is an excerpt from a scene on the funeral day of a great mafia boss in which his successor Michael wants to make sure of the movement of the other mafia bosses (represented by the wind). The supposition is, therefore, that Michael does not know the other bosses' inten- 
tions or decisions. Moreover, in this novel this funeral day is set as a turning point from which movement in some direction (represented by the wind) is initiated; therefore, the supposition is that he does not think that things have already started to go in a particular direction. From these, we consider not that the preliminary stage of the BGT-sentence in (37a) has already started to occur, but that it is starting to occur from that day on. Here, the subject is inanimate and the infinitive is a verb of activity. Thus, this BGTsentence is taken as an instance of non-intentional use of BGT-sentences of Stage B.

Let me next consider Stage C. This stage includes the predictive and the simple-future use of BGT-sentences. The two uses are defined in the following manner (these definitions apply not only to BGT-sentences but also to $\mathrm{W}$-sentences). The predictive use can be paraphrased as "I predict that ..." (Coates (1983: 179)), accompanying "a sense of expectation based on deductive or inductive reasoning" (Declerck (2006: 103)), and usually refers to the future, but can in principle refer to the present and the past. ${ }^{13}$ The propositional content of a sentence with this use must be worth evaluating or predicting subjectively. On the other hand, the simple-future use is "the least subjectified" (Declerck (2006: 103)), and the occurrence of the target situation itself is not subject to the speaker's judgment/evaluation or intention/decision or a combination of those (cf. Kashino (1999: 40)).

The temporal structure of Stage C of BGT-sentences is similar to that of the predictive and simple-future use of W-sentences schematized in Figure 3 (i) above, which reflects the meanings of the (epistemic) modal will and the bare infinitive, namely a present prediction with no ongoing process or a very schematic situation related to $E_{1}$, and the future occurrence or existence of a target situation related to $E_{2}$ (see Wada (2001a: Ch. 7) for details). Thus, in Stage C, we find it impossible for the preliminary stage to be interpreted as a process leading up to the occurrence of a future situation; since this stage excludes the process part, the coda part thus is also excluded in that the existence of the coda presupposes the process leading up to it. The onset part in Figure 6 (ii) above functions merely as a reference point or time of orientation in my terms. In order to preserve a posterior

13 The predictive use of BGT-sentences in my sense is different from the same term used in Kashino (1999: 59) and many other previous studies in that the former is restricted only to a pure prediction, i.e. a prediction without an ongoing process, while the latter is a cover term for uses other than the intentional use (including the use expressing a prediction with an ongoing process). 
relationship of $E_{2}$ with respect to $E_{1}$, at least some kind of future orientation has to be kept. I assume that this kind of future orientation exists in the mind of the speaker, which is symbolized by the broken arrow in Figure 6 (ii). This future orientation is supported by the fact that BGT-sentences in the present tense form necessarily refer to the future, and never refer to the present or the past (relative to speech time), as below. ${ }^{14}$

a. *Mary is going to be at home now.

b. *They are going to have arrived two hours ago.

Now, let me state when the temporal structure of Stage $C$ is reserved for the predictive use and when it is reserved for the simple-future use. As we saw above, in this stage only the onset part is represented by be going to and has come to function merely as a time of orientation. In the default case, modality holds at the time of utterance, and independent clauses usually accompany modality holding at speech time (now). Therefore, with the temporal structure of Stage C of BGT-sentences in the present tense form in independent clauses, the onset part is usually accompanied by a prediction when predictive modality holds at speech time. In this case, the temporal structure of Stage C represents the predictive use.

However, when a future situation is considered to occur as a matter of course without the speaker's evaluation and/or intention involved, for example, in the case of someone becoming a certain age, the degree of prediction at speech time is at a minimum. Therefore, the onset part is not accompanied by a prediction. To illustrate this case, consider (39):

(39) I'm going to be forty in a few years.

(Sheldon (1983: 204)) Since humans age as the years pass, the degree of prediction is at a minimum. This is a typical case of the simple-future use.

In this connection, Fujimoto (2007) addresses Wada (2002) to criticize the latter's explanation for the simple-future use of BGT-sentences. He claims that not only BGT-sentences of Stage B but also BGT-sentences of Stage $\mathrm{C}$ do not exist in present-day English, and criticizes what he considers to be Wada's (2002) conclusion, namely that the simple-future use of BGT-

14 It is stated in Wada (2001a: Ch. 7) that the temporal structure of Stage C of BGTsentences and that of the predictive and the simple-future use of W-sentences in Figure 3 (i) are different in that the former shows the so-called subjective future orientation (or the trace of the ongoing process), while the latter need not. For this reason, W-sentences can refer to the present and the past as well as the future, as illustrated by (i):

(i) a. Mary will be at home now.

b. They will have arrived two hours ago. 
sentences of Stage $\mathrm{C}$ has the same meaning as that of $\mathrm{W}$-sentences. (In the following discussion, I will ignore the intentional use of BGT- and Wsentences.)

However, Fujimoto's claim is based on a misunderstanding of Wada's explanation. First of all, in fact it is never stated in Wada (2002) that the simple-future use of BGT-sentences has the same temporal structure or meaning as the simple-future use of $\mathrm{W}$-sentences. On the contrary, the acceptability difference of BGT- and W-sentences concerning time reference (e.g. (38) vs. (i) in note 14) is pointed out in Wada (2001a: Ch. 7), which implies that the simple-future use and the predictive use of BGTsentences are slightly different in temporal structure from those of $\mathrm{W}$-sentences. Therefore, his criticism on this point is not appropriate.

Second, Fujimoto regards the BGT-sentence in (37b) as an instance of encroaching on the semantic range of $\mathrm{W}$-sentences, which is also a misunderstanding. As is evident from the temporal structure in Figure 6 (i) above, this BGT-sentence still has internal structure and thus shows a different temporal structure from that of $\mathrm{W}$-sentences in Figure 3 (i) above.

Furthermore, Fujimoto's alternative explanation is not tenable. Following Thomson and Martinet (1986) and Declerck (1991), he claims that with W-sentences, the speaker makes a prediction about the future subjectively, while with BGT-sentences, the speaker makes a prediction about the future on the basis of objective evidence. On this basis, he explains the acceptability difference in (40) in the following manner.

(40) My babe-in-arms \{will/??is going to be fifty nine on my eighty ninth birthday ... the year two thousand when I shall be ninety.

(Palmer (1988: 148))

This sentence indicates the following: the subject's referent is a little baby and has to live long to be 59; since s/he has not lived long, nobody knows whether $\mathrm{s} / \mathrm{he}$ can live that long. These suggest that there is not enough objective evidence for the baby's being able to reach that age. Hence, the use of the BGT-sentence is awkward, but the use of the W-sentence is natural.

Similarly, Fujimoto explains the use of the BGT-sentence in (39) above in the following manner. This sentence indicates that the speaker is approaching and will become forty in a couple of years. Therefore, there is enough objective evidence for her being able to reach the age in question; hence, the BGT-sentence is chosen.

This line of reasoning predicts that $\mathrm{W}$-sentences are used when there is little objective evidence that shows a person can reach a particular age. This is not the case, however. 
(41) Mr Robinson nodded and sipped at his drink. 'Ben?' he said. 'How old are you now?' 'Twenty. I'll be twenty-one next week.'

(Webb (1968: 25))

In (41), Ben uses the W-sentence to answer Mr Robinson's question. In this context, he is not considering committing suicide or having a fatal disease; moreover, reaching the target age comes in the very near future. That is, the evidence for a prediction about the future situation is sufficiently objective. Therefore, Fujimoto's reasoning cannot explain why the W-sentence is used here, and at the same time, cannot explain why the BGT-sentence is not chosen here.

To explain cases like this, Fujimoto argues that whether the speaker uses BGT-sentences or W-sentences is based on his/her subjective judgment, but not on whether objective evidence really exists or not. In his opinion, although sentence (42) - uttered in a scene in which the addressee is fatally injured-indicates that, objectively speaking, the evidence for the prediction about the addressee's being OK does not exist, this speaker behaves as if there were enough objective evidence, perhaps because she wants to reassure the addressee.

(42) Oh, oh. Baby, hold on. You're gonna be okay. Somebody! Somebody! Somebody help us.

Fujimoto (personal communication) argues that a case like the $\mathrm{W}$-sentence in (41) can also be explained in a similar way. However, such an account is not explanatory because it is not falsifiable. What is worse, the BGTsentence in (42) and one more sentence which he presents in his paper (i.e., Today's gonna be a less bad day) are concerned with topics that can be predicted subjectively or evaluated by the speaker. They are not instances of the simple-future use in Wada's sense.

From the observations made above, not only does Fujimoto's criticism of Wada's arguments for the simple-future use of BGT-sentences miss the point, but also his alternative account cannot explain the linguistic facts. I contend that the two variants of the temporal structure of BGT-sentences (the variants of Stage B and Stage C) are necessary. ${ }^{15}$

Let me now touch briefly on whether the temporal structure of PPF-

15 In my analysis, the reason why some native speakers hesitate or refuse to allow the use of BGT-sentences in the simple-future context (e.g. (40)) is that for such a speaker, the temporal structure of Stage $\mathrm{C}$ is not established in his/her mind. This is another merit of my approach. 
sentences has variants. I argue that PPF-sentences have only the single temporal structure, the one in Figure 4 (iii) above. The reasons for this argument are as follows. First, the temporal structures in Figures 4 (i) and (ii) above show present time reference. Second, the temporal structure of PPF-sentences has already emerged through grammaticalization from the basic structure of present progressive sentences expressing progressive aspect. Third, and most importantly, we cannot completely eliminate the backgrounded preliminary stage from the temporal structure of PPF-sentences in Figure 4 (iii) because it is a bridge between the present and the future, and the temporal structure without it cannot guarantee the tense structure of the present participle, namely a simultaneous relationship between the event time and the time of orientation (in this case, the event time of the progressive be). Therefore, PPF-sentences must have a temporal structure like the one in Figure 4 (iii).

\subsection{Explanation}

\subsubsection{Stative Verbs Expressing Permanent States}

I can now explain the two remaining problems mentioned at the beginning of this section. I will begin with the issue of compatibility of PPFand BGT-sentences with stative verbs. As we saw, it is generally stated in the literature that stative verbs expressing permanent states cannot occur in PPF-sentences. This is exemplified by the following sentences:

(43) a. Their new house is going to look over the river. $(=(10 \mathrm{a}))$

b. *Their new house is looking over the river. $(=(10 b))$

(44) a. *I'm knowing the answer tomorrow.

(Prince (1982: 455))

b. I'm going to know the answer tomorrow. ${ }^{16}$

The reason why stative verbs cannot occur in PPF-sentences is explained straightforwardly in my analysis. As is clear from the temporal structure in Figure 4 (iii), the outcome stage is actualized as a result of the end of the preliminary stage. This requires that the situation associated with the outcome stage be either a non-stative or a change-of-state type. By contrast, in the case of the temporal structure of BGT-sentences of Stage C in Figure 6 (ii), there is no preliminary stage leading to the occurrence of the infinitive's situation; the onset part of the be going to situation functions merely as time of orientation for calculating the position of event time of the infinitive. Hence, this temporal structure does not require the infinitive's situa-

\footnotetext{
${ }^{16}$ I owe this sentence to an anonymous reviewer.
} 
tion to be non-stative. ${ }^{17}$

\subsubsection{Prediction}

Finally, I will briefly explain the reason why PPF-sentences cannot receive a predictive reading, while BGT-sentences can, by considering (15a, b), repeated here as $(45 \mathrm{a}, \mathrm{b})$ :

(45) a. *You're breaking that chair if you're not careful.

b. You are going to break that chair if you're not careful.

It is often said that W-sentences co-occur with if-clauses expressing futurate open conditions, but BGT-sentences cannot do so because the present orientation of BGT-sentences is contradictory to the future orientation of futurate open conditionals (cf. Leech (2004: 60)). ${ }^{18}$ Nevertheless, sentence (45b) is acceptable (for many native speakers). This suggests that for them, the BGT-sentence in (45b) does not have the basic temporal structure in Figure 5. Because this context brings about a predictive reading of the main clause (see section 3.1.3), this BGT-sentence is assumed to have the temporal structure of Stage $\mathrm{C}$ of the predictive use, which is more similar to the temporal structure of the predictive use of $\mathrm{W}$-sentences. Thus, my

17 Prince (1982: 455) states that stative verbs can occur in SPF-sentences, as exemplified by I know the answer tomorrow, when the sentences receive an inchoative reading. In this reading, know expresses a kind of change of state. Wada (2001b) accepts Goldsmith and Woisetschlaeger's (1982) argument that the temporal structure of SPFsentences reflects the properties of "structural description," which is schematically represented as below.

A:

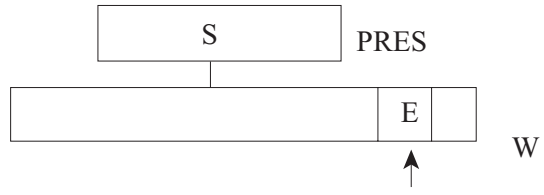

$\mathrm{TF}$

W symbolizes the world (structure) which exists at or around speech time. This figure suggests that one event which is assumed to constitute the world (structure) is certain to occur in the future. This shows that the degree of certainty of the occurrence of the future event is very high (or higher than in the case of PPF-sentences (Prince (1982: 453)), and their main semantic interpretations, such as schedule and inevitability, come from this high degree of certainty, which is attributed to the temporal structure of SPF-sentences.

18 It is usually noted in the literature that BGT-sentences co-occur with if-clauses if the condition is a present one, i.e., if present circumstances are mentioned, as in:

(i) We're going to find ourselves in difficulty if we go on like this.

(Leech (2004: 60))

I am ignoring conditionals like this in the main text. 
analysis explains why BGT-sentences can co-occur with futurate open conditionals. By contrast, PPF-sentences have only the single temporal structure shown in Figure 4 (iii), where the backgrounded preliminary stage indicates the present evidence for the occurrence of a future situation. Thus, PPFsentences cannot co-occur with futurate open conditionals, in that the present orientation of PPF-sentences is contradictory to the future orientation of those conditionals. ${ }^{19}$

\section{Concluding Remarks}

In this paper, I have developed the temporal structures of PPF- and BGTsentences within the framework of Wada (2001a). The characteristics of the two sentence types can be summarized as follows. PPF-sentences include an extended process consisting of the backgrounded preliminary stage and the outcome stage, both of which are associated with $E_{2}$, the event time of the present-participle situation; the schematic situation represented by progressive $b e$, associated with $\mathrm{E}_{1}$, indirectly implies the existence of that backgrounded preliminary stage. BGT-sentences include two different independent situations, i.e. the be going to situation corresponding to the preliminary stage, and the infinitive's situation corresponding to the outcome stage; the former is associated with $E_{1}$ and the latter with $E_{2}$. PPF-sentences have only the single temporal structure shown in Figure 4 (iii), while BGTsentences have at least the three structures shown in Figures 5, 6 (i), and 6 (ii). I have demonstrated that these characteristics provide a basis for explaining the three points in (18) systematically and from a unified point of view.

19 An anonymous reviewer has pointed out to me that for him/her, the PPF-sentences as well as the W- and BGT-sentences in (i) and (ii) are acceptable, which might contradict my claim in the main text.

(i) If you break that chair, \{a. you're paying/b. you're gonna pay/c. you'll pay for it.

(ii) If he brings a gun in here, \{a. I'm calling/b. I'm gonna call/c. I'll call $\}$ the police.

I tentatively argue that those who judge (ia) and (iia) to be acceptable emphasize the posterior relationship between $\mathrm{E}_{1}$ and the focused part of $\mathrm{E}_{2}$, de-emphasizing the existence of the backgrounded preliminary stage in the use of PPF-sentences; therefore, for them the temporal structure in Figure 4 (iii) is much more similar to that of W-sentences in Figure 3 (i). This is in keeping with Hopper and Traugott's (1993: 17) statement that language development or grammaticalization is an ongoing process. 
Finally, a major implication of these results is that Reichenbachian approaches to tense, based on the three time points $\mathrm{S}, \mathrm{R}$, and $\mathrm{E}$ (speech, reference, and event times), are too simple to model the tense phenomena dealt with in this paper. Another important implication is that non-compositional approaches cannot account for these tense phenomena in a way similar to how my compositional approach accounts for them with the temporal structures of PPF- and BGT-sentences in this paper.

\section{REFERENCES}

Binnick, Robert I. (1971) "Will and Be Going To," CLS 7, 40-52.

Binnick, Robert I. (1972) "Will and Be Going To II," CLS 8, 3-9.

Brisard, Frank (2001) "Be Going To: An Exercise in Grounding," Journal of Linguistics $37,251-285$.

Coates, Jennifer (1983) The Semantics of the Modal Auxiliaries, Croom Helm, London.

Crowther, Jonathan, et al. (1995) Oxford Advanced Learner's Dictionary, 5th ed., Oxford University Press, Oxford.

Dancygier, Barbara (1998) Conditionals and Prediction: Time, Knowledge and Causation in Conditional Constructions, Cambridge University Press, Cambridge.

Declerck, Renaat (1991) A Comprehensive Descriptive Grammar of English, Kaitakusha, Tokyo.

Declerck, Renaat (in cooperation with Susan Reed and Bert Cappelle) (2006) The Grammar of the English Verb Phrase Volume I: The Grammar of the English Tense System, Mouton de Gruyter, Berlin and New York.

Duffley, Patrick J. (1992) The English Infinitive, Longman, London.

Fujimoto, Shigeyuki (2007) "Will to Be Going To Saiko (A Reconsideration of Will and Be Going To)," Eigo to Bunpo to (English and Grammar), ed. by Akira Mizokoshi, Hiromi Onozuka, Shigeyuki Fujimoto, Nobuhiro Kaga, Toshiaki Nishihara, Makoto Kondo and Michiyo Hamasaki, 207-218, Kaitakusha, Tokyo.

Goldsmith, John and Erich Woisetschlaeger (1982) "The Logic of the English Progressive," Linguistic Inquiry 13, 79-89.

Haegeman, Liliane (1989) "Be Going To and Will: A Pragmatic Account," Journal of Linguistics 25, 291-317.

Hirtle, Walter H. and Violetta N. Curat (1986) "The Simple and the Progressive: 'Future' Use," Transactions of the Philological Society 1986, 42-84.

Hopper, Paul J. and Elizabeth C. Traugott (1993) Grammaticalization, Cambridge University Press, Cambridge.

Huddleston, Rodney (1977) "The Futurate Construction," Linguistic Inquiry 8, 730-736.

Janssen, Theo A. J. M. (1996) "Tense in Reported Speech and Its Frame of Refer- 
ence," Reported Speech, ed. by Theo A. J. M. Janssen and Wim van der Wurff, 237-259, John Benjamins, Amsterdam and Philadelphia.

Kashino, Kenji (1999) Tensu to Asupekuto no Goho (Usage of Tense and Aspect), Kaitakusha, Tokyo.

Leech, Geoffrey (1987) Meaning and the English Verb, 2nd ed., Longman, London.

Leech, Geoffrey (2004) Meaning and the English Verb, 3rd ed., Longman, London.

McIntosh, Angus (1966) "Predictive Statements," In Memory of J. R. Firth, ed. by Charles E. Bazell, John C. Catford, Michael A. K. Halliday and Robert H. Robins, 303-320, Longman, London.

Nakau, Minoru (1994) Ninchi Imiron no Genri (Principles of Cognitive Semantics), Taishukan, Tokyo.

Nesselhauf, Nadja (2007) “The Spread of the Progressive and Its 'Future' Use," English Language and Linguistics 11, 191-207.

Nicolle, Steve (1998) "Be Going To and Will: A Monosemous Account," English Language and Linguistics 2, 223-243.

Palmer, Frank R. (1988) The English Verb, 2nd ed., Longman, London.

Prince, Ellen (1982) "The Simple Futurate: Not Simply Progressive Futurate Minus Progressive," CLS 18, 453-465.

Sinclair, John et al. (2006) Collins COBUILD Advanced Learner's English Dictionary, 5th ed., HarperCollins, Glasgow.

Smith, Carlota (1981) "The Futurate Progressive: Not Simply Future + Progressive," CLS 17, 369-382.

Swan, Michael (1995) Practical English Usage, 2nd ed., Oxford University Press, Oxford.

Sweetser, Eve (1988) "Grammaticalization and Semantic Bleaching," BLS 14, 389405.

Thomson, Audrey J. and Agnes V. Martinet (1986) A Practical English Grammar, 4th ed., Oxford University Press, Oxford.

Traugott, Elizabeth C. and Richard B. Dasher (2002) Regularity in Semantic Change, Cambridge University Press, Cambridge.

Traugott, Elizabeth C. and Bernd Heine, eds. (1991) Approaches to Grammaticalization, John Benjamins, Amsterdam.

Wada, Naoaki (1996) "Does Doc Brown Know Which Expression Takes Us Back to the Future: Be Going To or Will?" English Linguistics 13, 169-198.

Wada, Naoaki (2001a) Interpreting English Tenses: A Compositional Approach, Kaitakusha, Tokyo.

Wada, Naoaki (2001b) "Eigo no Tanjungenzaikei ni Taisuru Toitsuteki Setsumei (A Unified Account of the Simple Present Tense Form in English)," Tsukuba Eigo Kyoiku 22, 1-16.

Wada, Naoaki (2002) "Iwayuru Be Going To no Tanjunmirai Yoho ni Tsuite (On the So-called Simple-Future Use of Be Going To)," Eigo Seinen 147.11, 30-31.

Wekker, Charles H. (1976) The Expression of Future Time in Contemporary British English, North-Holland Publishing Company, Amsterdam.

Wierzbicka, Anna (1988) The Semantics of Grammar, John Benjamins, Amsterdam. 


\section{Sources of Examples}

Gardner, Craig S. (1990) Back to the Future Part III, Berkley Books, New York. Puzo, Mario (1969) The Godfather, Mandarin, London.

Sheldon, Sidney (1983) Master of the Game, Pan Books, London.

Webb, Charles (1968) The Graduate, Penguin Books, London.

[received September 14 2008, revised and accepted January 26 2009]

Doctoral Program in Literature and Linguistics

University of Tsukuba

1-1-1 Ten-nodai, Tsukuba-shi

Ibaraki 305-8571

e-mail: naoaki@lingua.tsukuba.ac.jp 